\title{
Expression of Bone Morphogenetic Proteins in Multiple Sclerosis Lesions
}

\author{
Carme Costa ${ }^{* \dagger}$ Herena Eixarch, ${ }^{* \dagger}$ Elena Martínez-Sáez, ${ }^{\dagger \dagger}$ Laura Calvo-Barreiro, ${ }^{* \dagger}$ Maite Calucho, ${ }^{* \dagger}$ Zoraida Castro, ${ }^{* \dagger}$ \\ Arantxa Ortega-Aznar, ${ }^{\dagger \dagger}$ Santiago Ramón y Cajal, ${ }^{\dagger \ddagger}$ Xavier Montalban, ${ }^{* \dagger \S}$ and Carmen Espejo ${ }^{* \dagger}$
}

From the Neurology-Neuroimmunology Department,* Multiple Sclerosis Centre of Catalonia (Cemcat), Vall d'Hebron Research Institute (VHIR), Vall d'Hebron University Hospital, Barcelona, Spain; Autonomous University of Barcelona, ${ }^{\dagger}$ Barcelona, Spain; the Pathology Department, ${ }^{\ddagger}$ Vall d'Hebron University Hospital, Autonomous University of Barcelona, Spanish Biomedical Research Network Centre in Oncology (CIBERONC), Barcelona, Spain; and the Division of Neurology, ${ }^{\complement}$ BARLO Multiple Sclerosis Centre, St. Michael's Hospital, University of Toronto, Toronto, Ontario, Canada

\author{
Accepted for publication \\ November 2, 2018. \\ Address correspondence to \\ Carmen Espejo, Ph.D., Servei \\ de Neurologia- \\ Neuroimmunologia, Centre \\ d'Esclerosi Múltiple de \\ Catalunya, Vall d'Hebron \\ Institut de Recerca, Hospital \\ Universitari Vall d'Hebron, \\ Ps Vall d'Hebron 119-129, \\ Collserola Building, 08035 \\ Barcelona, Spain. E-mail: \\ carmen.espejo@vhir.org.
}

\begin{abstract}
Bone morphogenetic proteins (BMPs) are secreted proteins that belong to the transforming growth factor- $\beta$ superfamily. In the adult brain, they modulate neurogenesis, favor astrogliogenesis, and inhibit oligodendrogenesis. Because BMPs may be involved in the failure of remyelination in multiple sclerosis (MS), we characterized the expression of BMP-2, BMP-4, BMP-5, and BMP-7; BMP type II receptor (BMPRII); and phosphorylated SMAD (PSMAD) 1/5/8 in lesions of MS and other demyelinating diseases. A total of 42 MS lesions, 12 acute ischemic lesions, 8 progressive multifocal leukoencephalopathy lesions, and 10 central nervous system areas from four nonneuropathological patients were included. Lesions were histologically classified according to the inflammatory activity. The expression of BMP-2, BMP-4, BMP-5, BMP-7, BMPRII, and pSMAD1/5/8 was quantified by immunostaining, and colocalization studies were performed. In MS lesions, astrocytes, microglia/macrophages, and neurons expressed BMP-2, BMP-4, BMP-5, and BMP-7; BMPRII; and PSMAD1/5/8. Oligodendrocytes expressed BMP-2 and BMP-7 and PSMAD1/5/8. The percentage of cells that expressed BMPs, BMPRII, and pSMAD1/5/8 correlated with the inflammatory activity of MS lesions, and changes in the percentage of positive cells were more relevant in MS than in other white matter-damaging diseases. These data indicate that BMPs are increased in active MS lesions, suggesting a possible role in MS pathogenesis. (Am J Pathol 2019, 189: 665-676; https://doi.org/10.1016/j.ajpath.2018.11.007)
\end{abstract}

Bone morphogenetic proteins (BMPs) are secreted factors that are members of the transforming growth factor- $\beta$ superfamily. Approximately 20 BMPs have been described and classified into subgroups according to sequence similarities and functions. ${ }^{1}$ BMP receptors are transmembrane serine/threonine kinase receptors that are classified into two subgroups: types I and II. ${ }^{2}$ As dimeric ligands, BMPs bind to type II dimeric receptors and subsequently recruit type I dimeric receptors to form a heterotetrameric receptor complex that signals through SMAD $1 / 5 / 8 .^{3}$ Phosphorylated SMAD (pSMAD) 1/5/8 translocates into the nucleus and regulates the transcription of BMP-dependent genes. ${ }^{4}$ Their role in the developing central nervous system (CNS) has been well studied, ${ }^{5}$ and it is known that BMPs are involved in many physiological processes in adults. BMPs are morphogens that are mainly involved in the regulation of cell lineage fate. In the CNS, BMPs favor astrogliogenesis as they simultaneously impede the differentiation and maturation of oligodendrocyte progenitor cells (OPCs) into mature oligodendrocytes, thus disturbing myelination. ${ }^{6,7}$

\footnotetext{
Supportedby the Fondo de Investigación Sanitaria (FIS) grant PI12/ 02144 (C.E.); Instituto de Salud Carlos III, Ministry of Economy, Industry and Competitivity, Spain; the Fundación Salud 2000-Merk Serono, Spain grant SERONO-2013-04 (C.E.); the Red Española de Esclerosis Múltiple grant RD12/0032 (X.M.), sponsored by the FIS; and the Ajuts per donar Suport als Grups de Recerca de Catalunya grant 2014 SGR 1082 (X.M.), sponsored by the Agència de Gestió d'Ajuts Universitaris i de Recerca, Generalitat de Catalunya, Spain.

C.C. and H.E. contributed equally to this work.

Disclosures: X.M. reports personal fees from Actelion, Bayer, Biogen, Celgene, Hoffmann-La Roche, Merck, Novartis, Oryzon Genomics, SanofiGenzyme, and Teva Pharmaceutical, not relevant to this work.
} 
Table 1 Characteristics of MS Brain Blocks

\begin{tabular}{lllllllll}
\hline Patient no. & Sex & $\begin{array}{l}\text { Age, } \\
\text { years }\end{array}$ & $\begin{array}{l}\text { Postmortem } \\
\text { delay, hours }\end{array}$ & MS type & CHIAs, $n$ & CLIAs, $n$ & CIAs, $n$ & Cause of death \\
\hline EM1 & F & 62 & 17 & RRMS & 0 & 0 & 1 & Pulmonary thromboembolism \\
EM2 & M & 45 & 10 & RRMS & 0 & 3 & 3 & Acute myocardial infarct \\
EM3 & $\mathrm{F}$ & 50 & 20 & RRMS & 4 & 2 & 2 & Bronchopneumonia \\
EM4 & $\mathrm{F}$ & 46 & $<24$ & RRMS & 4 & 1 & 1 & Septic endocarditis (Escherichia coli) \\
EM5 & $\mathrm{M}$ & 47 & $<24$ & RRMS & 17 & 3 & 1 & Pulmonary thromboembolism \\
\hline
\end{tabular}

F, female; M, male; CHIA, chronic MS lesion with high inflammatory activity; CIA, chronic inactive MS lesion; CLIA, chronic MS lesion with low inflammatory activity; MS, multiple sclerosis; RRMS, relapsing-remitting MS.

Similarly, BMPs are up-regulated when demyelination occurs, favoring the differentiation of astrocytes over oligodendrocytes, probably hindering the repair of the damaged tissue. ${ }^{8}$ BMPs are essential to maintain the undifferentiated state of neural stem cells within the adult CNS. In fact, a precise regulation of BMP signaling is necessary to maintain the balance between quiescence/self-renewal and proliferation/differentiation processes to ensure appropriate neurogenesis throughout adulthood. ${ }^{9}$

Because BMPs are important in demyelination, oligodendrocyte differentiation, and maturation, as well as in the control of the neural stem cell pool, their signaling pathway may represent a putative therapeutic target for multiple sclerosis (MS), a chronic, demyelinating, immune-mediated disease of the CNS. ${ }^{10}$ Alterations in the CNS mainly (but not only) caused by the local immune response, including demyelination, axonal loss, and gliosis, clearly contribute to the neurodegeneration that leads to a chronic disability. ${ }^{11}$ Although there is evidence of extensive remyelination even in lesions of long-diseased MS patients, ${ }^{12,13}$ proper remyelination does not take place in a significant proportion of MS lesions. Data support that OPC migration and differentiation are impaired in MS, ${ }^{14}$ and that premyelinating oligodendrocytes are found in chronic lesions, although they are not capable of remyelinating. ${ }^{15}$ The cause of remyelination failure in MS is far from being understood; nevertheless, several hypotheses have been proposed, including the presence of inhibitory molecules in the microenvironment of the lesion that would impede the recruitment and differentiation of OPCs that promote tissue repair in the

Table 2 Antibodies and Conditions Used for Immunohistochemistry

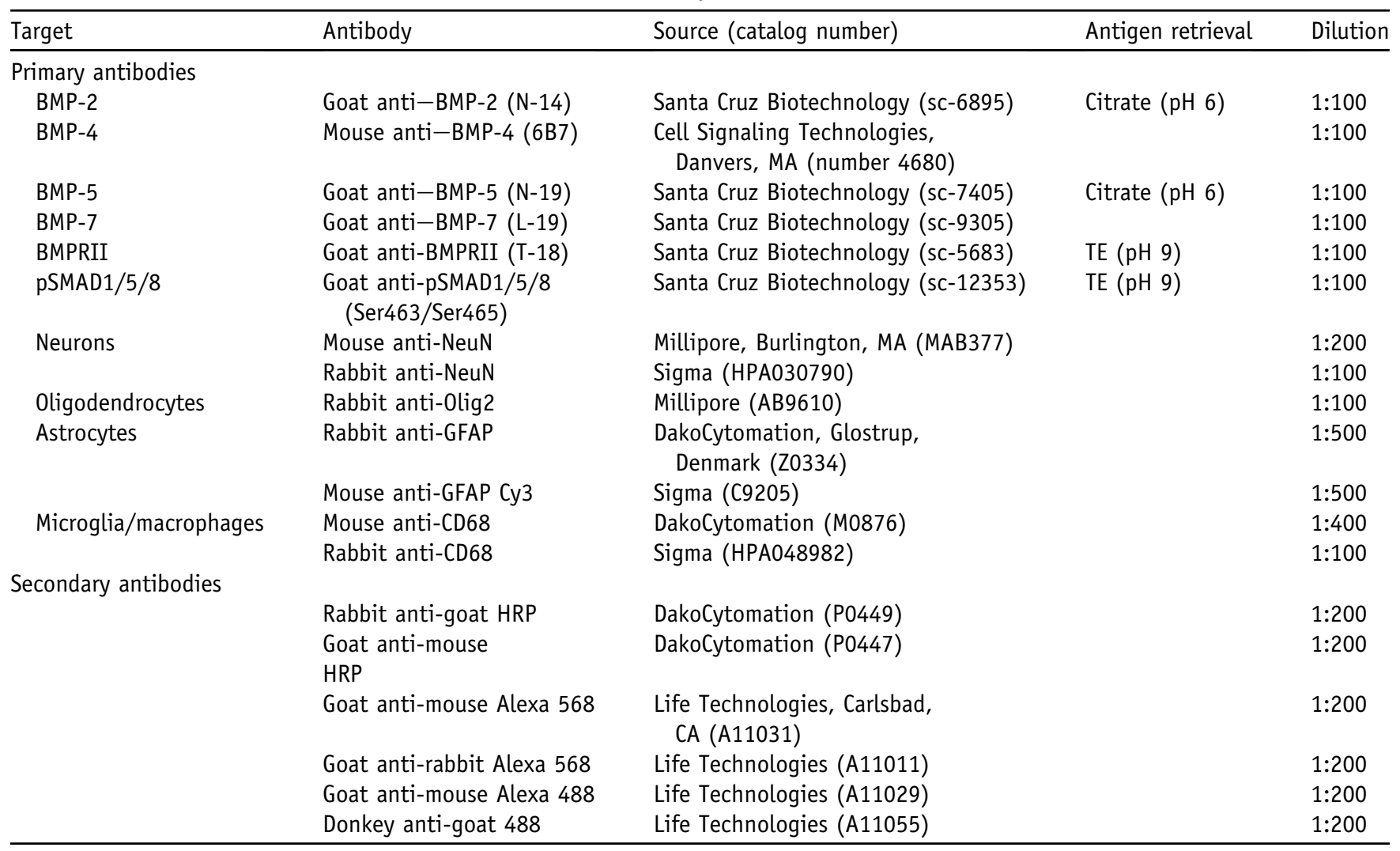

BMP, bone morphogenetic protein; BMPRII, BMP type II receptor; GFAP, glial fibrillary acidic protein; HRP, horseradish peroxidase; NeuN, neuronal nuclei; Olig2, oligodendrocyte transcription factor 2; PSMAD, phosphorylated SMAD. 


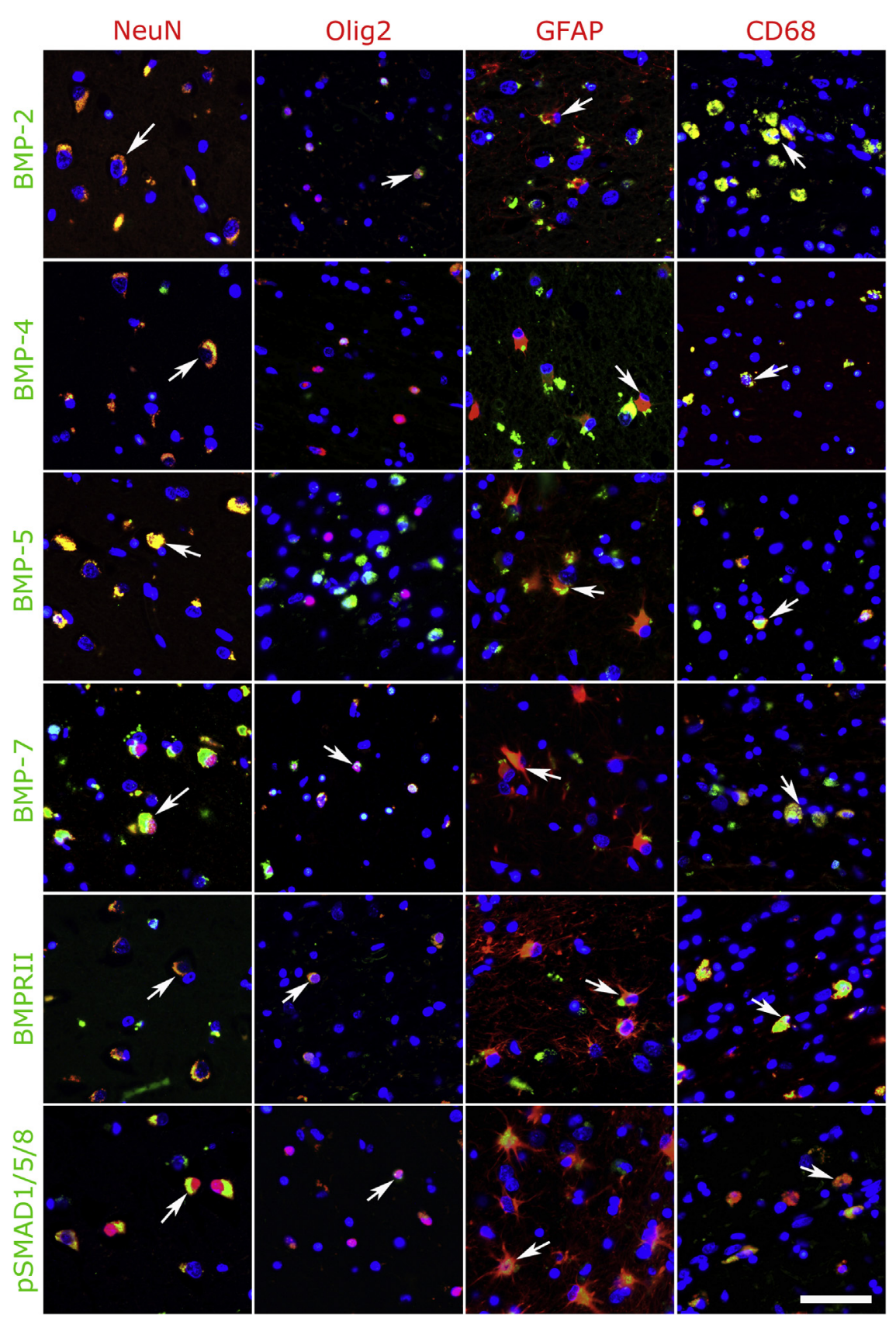

Figure 1 Characterization of bone morphogenetic proteins (BMPs), BMP type II receptor (BMPRII), and phosphorylated SMAD (pSMAD) 1/ $5 / 8$ in multiple sclerosis (MS) lesions. Micrographs of chronic with high inflammatory activity MS lesions showing the expression of BMP-2, BMP-4, BMP-5, and BMP-7, pSMAD1/5/8, and BMPRII (green) in different cell types (red): neurons (NeuN), oligodendrocytes (0lig2), astrocytes [glial fibrillary acidic protein (GFAP)], and macrophages (CD68). All images, except NeuN staining, were taken in the white matter at the margins of MS lesions where major inflammatory activity is found. Neurons were detected in the gray matter adjacent to the lesion. Arrows indicate colocalization of immunofluorescence staining. Scale bar $=100 \mu \mathrm{m}$ (all images). Original magnification, $\times 40$ (all images). NeuN, neuronal nuclei; Olig2, oligodendrocyte transcription factor 2.

damaged areas. ${ }^{16} \mathrm{We}$, therefore, hypothesize that BMPs may contribute to the pathogenesis of MS. In this study, we describe the expression of BMPs in chronic MS lesions and determine whether their expression is relevant in MS compared with other demyelinating diseases.

\section{Materials and Methods}

Tissue Samples

Seventy-two CNS areas from 21 patients with neuropathological postmortem study, performed between 1992 and 2009 at the Pathology Department, Vall d'Hebron
University Hospital, were retrospectively selected: 42 demyelinating lesions from five relapsing-remitting MS patients (Table 1), 12 lesions from eight patients with acute cerebral infarct (AI), 8 lesions from four patients with progressive multifocal leukoencephalopathy (PML), and 10 normal-appearing white matter and normal-appearing gray matter (NAGM) areas from four nonneurologic control (NNC) patients. The autopsy delay was $<24$ hours, and all samples were fixed in $10 \%$ buffered formalin for 4 weeks and embedded in paraffin.

The study was approved by the Clinical Research Ethics Committee of Vall d'Hebron University Hospital [PR(AG) 304/2012]. 


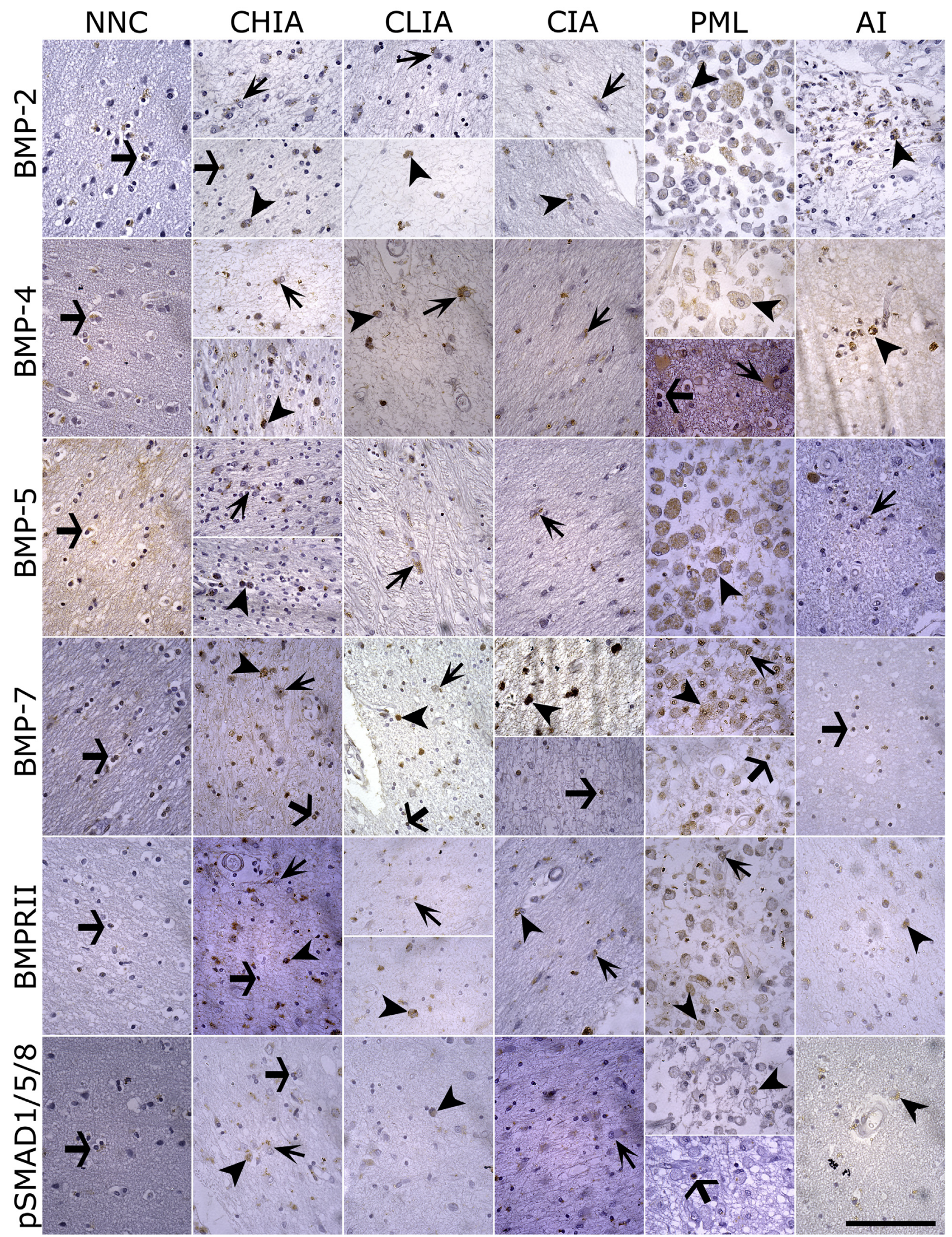




\section{Histopathological Characterization of Demyelinated Lesions and Classification of MS Lesions}

Formalin-fixed, paraffin-embedded blocks were cut into serial sections ( $4 \mu \mathrm{m}$ thick) and stained with hematoxylin and eosin and Klüver-Barrera for inflammation and demyelination assessment, respectively. MS lesions were subsequently classified into chronic with high inflammatory activity (CHIA; $n=25$ ), chronic with low inflammatory activity (CLIA; $n=9$ ), and chronic inactive (CIA; $n=8) .{ }^{17}$ Briefly, CHIA lesions were hypercellular, with perivascular and lesion border inflammatory infiltration and active demyelination. CLIA lesions showed hypocellularity with scarce perivascular inflammatory infiltration and no active demyelination. CIA lesions showed neither inflammation nor active demyelination. AI lesions included the acute ischemic core with pale neuropil and acute ischemic changes in neurons (piknotic nuclei, eosinophilic cytoplasms) and the penumbra area with vacuolation of the white matter and axonal swelling, to compare affected and relatively preserved areas. PML changes consisted of demyelination with myelin-containing macrophages, bizarre reactive astrocytes, and oligodendrocytes with enlarged glassy nuclei reflecting viral damage. Mild neuropathological changes were found in NNC, mainly represented by scarce perivascular macrophages, probably as a result of mild small-vessel disease. In MS, AI, and PML lesions, NAGM proximal to the lesion was studied; and in NNC, NAGM was studied.

\section{Immunohistochemistry}

Paraffin-embedded serial sections $(4 \mu \mathrm{m}$ thick) were deparaffinized in xylene and rehydrated in alcohol. Endogenous peroxidase activity was blocked with hydrogen peroxide (2\%; Sigma, St. Louis, MO) and methanol (70\%; Merck, Darmstadt, Germany) for $20 \mathrm{mi}-$ nutes. Antigen retrieval was performed in $10 \mathrm{mmol} / \mathrm{L}$ citrate $(\mathrm{pH}=6)$ or Tris-EDTA buffer $[1 \mathrm{~mol} / \mathrm{L}$ TrismaBase and $1 \mathrm{mmol} / \mathrm{L}$ EDTA (Sigma), pH 9] when needed (Table 2). Nonspecific protein binding was blocked with $2 \%$ bovine serum albumin (Sigma) in phosphate-buffered saline. Sections were incubated overnight at $4^{\circ} \mathrm{C}$ with the primary antibodies listed in Table 2 . Antibody binding was visualized with appropriate secondary antibodies (Table 2) and 3,3'diaminobenzidine (Sigma) as the chromogenic substrate. Negative controls consisting of tissue samples that underwent the same protocol but that were only immunostained with the appropriate secondary antibodies were included.

Double-immunofluorescence staining for BMP-2, BMP-4, BMP-5, and BMP-7; BMP type II receptor (BMPRII); pSMAD1/5/8; and different cell types, including oligodendrocytes, neurons, astrocytes, and microglia/macrophages, was performed. After deparaffinization, nonspecific protein binding was blocked with $2 \%$ bovine serum albumin and $0.3 \%$ Triton X-100 (Sigma) in phosphate-buffered saline solution. Antigen retrieval was performed in citrate buffer $(\mathrm{pH} 6)$ or TE buffer $(\mathrm{pH}$ 9) when needed (Table 2). Sections were incubated overnight at $4{ }^{\circ} \mathrm{C}$ with primary antibodies (Table 2). Appropriate secondary antibodies were incubated for 1 hour at room temperature (Table 2). Samples incubated with the secondary antibody were included as negative controls.

\section{Immunostaining Assessment}

Immunostaining of BMPs, BMPRII, and pSMAD1/5/8 was performed in NNC and MS, AI, and PML lesions. A neuropathologist (C.C.) blinded to the clinicopathological data evaluated immunostained slides. Cells expressing the different studied proteins were morphologically identified as microglia/macrophages, astrocytes, or oligodendrocytes in the white matter and as neurons in the NAGM proximal to the lesion. The percentage of total cells expressing BMP-2, BMP-4, BMP-5, and BMP-7; BMPRII; and pSMAD1/5/8 was assessed in the damaged white matter and, when available, in the NAGM proximal to the lesion. For each sample, BMP-, BMPRII-, and pSMAD1/5/8-positive cells were quantified in five fields of $0.0025 \mathrm{~mm}^{2}$. To avoid bias due to differences in cellularity, results are expressed as the percentage of positive cells for each staining over total cells in the field.

\section{Statistical Analysis}

Because of the heterogeneous variability of the data, statistical analyses were performed using nonparametric tests. The Kruskal-Wallis test was used to examine differences in expression between different disease conditions in the white and gray matter. Subsequently, Dunn test to adjust significance levels was applied to evaluate the pairwise comparisons of interest. ${ }^{18}$ Analyses were performed with R software version 3.2.3. ${ }^{19}$ The level of statistical significance was set at 0.05 . The data are expressed as the means $\pm \mathrm{SD}$.

Figure 2 Characterization of bone morphogenetic protein (BMP)-2, BMP-4, BMP-5, BMP-7, BMP type II receptor (BMPRII), and phosphorylated SMAD (pSMAD) $1 / 5 / 8$ in the white matter of the different disease conditions studied. The expression of BMP-2, BMP-4, BMP-5, and BMP-7, BMPRII, and pSMAD1/5/8 was assessed in the damaged white matter at the edge of the lesions in chronic multiple sclerosis (MS) lesion with high inflammatory activity (CHIA), chronic MS lesion with low inflammatory activity (CLIA), and chronic inactive (CIA) MS lesions, in acute infarct (AI) lesion, in progressive multifocal leukoencephalopathy (PML) lesions, and in normal-appearing white matter in nonneurologic control (NNC). Different cell types were morphologically identified. Arrows indicate positive immunostaining, arrowheads, microglia/macrophages; arrows with thick heads, astrocytes; arrows with thin heads, oligodendrocytes. Scale bar $=100 \mu \mathrm{m}$ (all images). Original magnification, $\times 40$ (all images). 
NNC

CHIA

CLIA

CIA

PML

AI

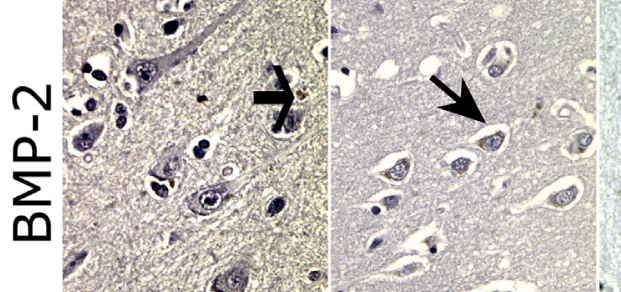

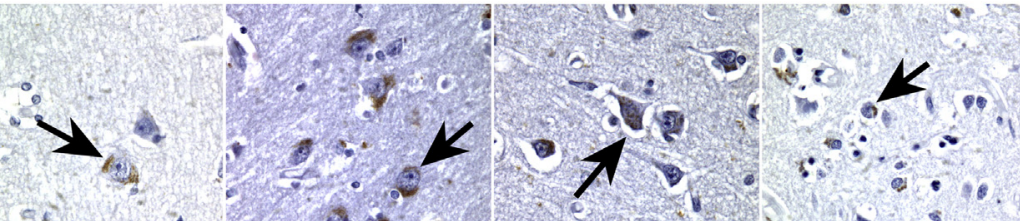

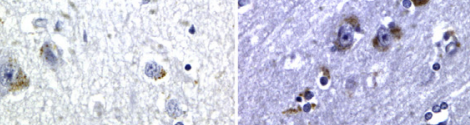

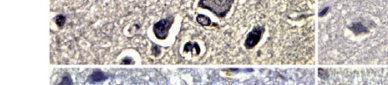

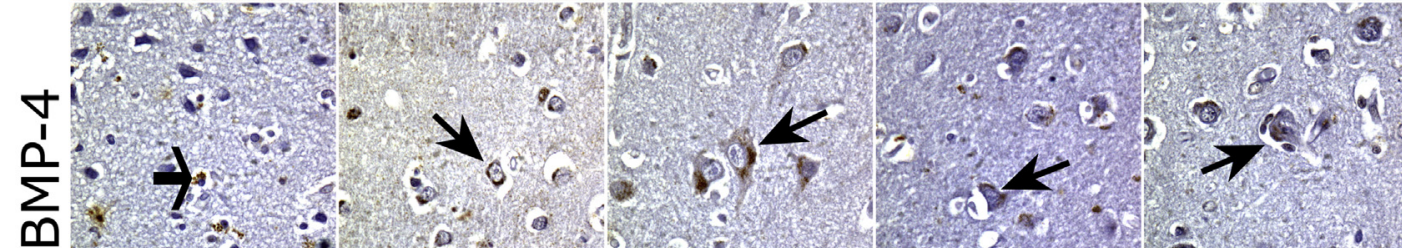

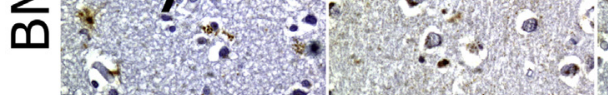

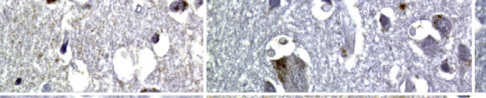

(9)

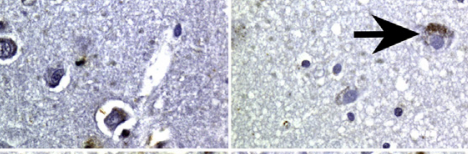

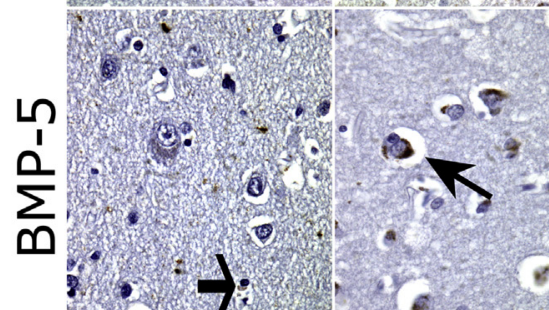

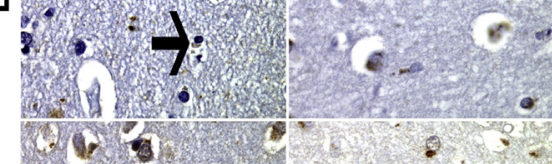

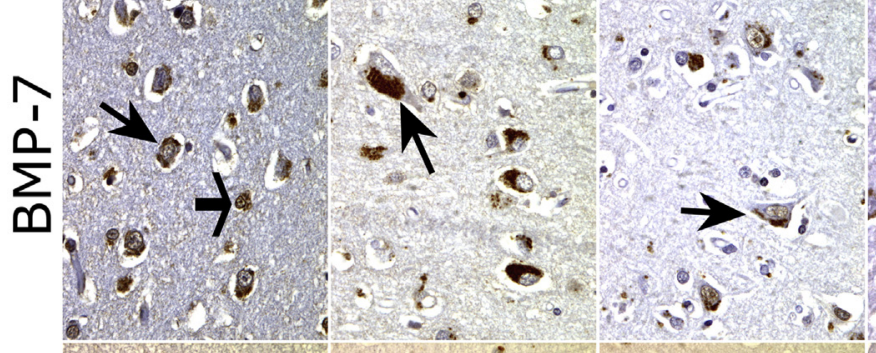

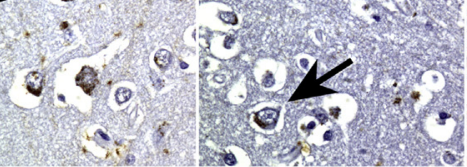

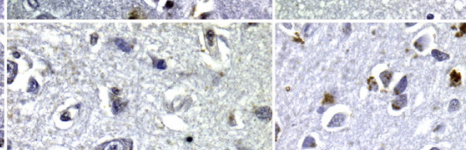

$\because n)^{2}$

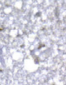




\section{Results}

BMPs, BMPRII, and PSMAD1/5/8 Are Expressed in Different Cell Types in MS Lesions

First, the expression of BMP-2, BMP-4, BMP-5, and BMP7; BMPRII; and the phosphorylated form of the cytoplasmic mediator SMAD1/5/8 was described through double immunostaining in CHIA MS lesions. At the margins of the lesion, astrocytes and microglia/macrophages expressed all BMPs, BMPRII, and pSMAD1/5/8 (Figure 1). On the other hand, oligodendrocytes showed only BMP-2 and BMP-7 expression, and BMPRII and pSMAD1/5/8 were also present, indicating that BMP signaling is active in this cell type (Figure 1). In the NAGM proximal to the lesion, the neurons expressed BMP-2, BMP-4, BMP-5, and BMP-7; BMPRII; and pSMAD1/5/8 (Figure 1). Negative controls showed no staining (Supplemental Figure S1), indicating that the immunoreactivity was specific for each primary antibody.

\section{Higher Expression of BMPs, BMPRII, and pSMAD1/5/8 in MS Lesions with High Inflammatory Activity}

The percentage of total BMP-2, BMP-4, BMP-5, and BMP7; BMPRII; and pSMAD1/5/8 expressing cells was studied in different types of MS lesions classified on the basis of their inflammatory and demyelination features as CHIA, CLIA, and CIA, as stated in Materials and Methods.

Morphologically identified microglia/macrophages and astrocytes in CLIA lesions showed expression of BMP-2, BMP-4, and BMP-7 and BMPRII. BMP-5 was observed only in astrocytes, pSMAD $1 / 5 / 8$ in the cytoplasm of microglia/macrophages, and BMP-7 in oligodendrocytes. In CIA MS lesions, BMP-2 and BMPRII were observed in astrocytes and microglia/macrophages, BMP-4, BMP-5, and pSMAD1/5/8 were detected in astrocytes, and BMP-7 was expressed only in the cytoplasm of oligodendrocytes and microglia/macrophages (Figure 2). In the NAGM proximal to the lesion, the same pattern of expression of BMPs, BMPRII, or pSMAD1/5/8 was observed in neurons in the different MS lesions (Figure 3). Negative controls showed no staining (data not shown), indicating that the immunoreactivity was specific for each primary antibody.

It was next determined whether the inflammatory activity in the MS lesion affected the percentage of expression of BMPs, BMPRII, and pSMAD1/5/8. In the damaged white matter, larger differences were observed when comparing
CHIA and CIA MS lesions. Percentage of BMP-2 $(23.77 \% \pm 8.10 \%$ versus $7.08 \% \pm 4.42 \% ; P<0.05)$, BMP-4 $(17.50 \% \pm 7.02 \%$ versus $6.29 \% \pm 5.15 \%$; $P<0.05), \quad$ BMP-7 $(46.44 \% \pm 12.65 \%$ versus $15.94 \% \pm 2.90 \% ; \quad P<0.05)$, and BMPRII $(24.92 \% \pm 9.26 \%$ versus $11.37 \% \pm 4.53 \% ; P<0.05)$ expressing cells was higher in CHIA compared with CIA MS lesions, whereas no differences in the percentage of cells expressing BMP-5 and pSMAD1/5/8 were observed (Figures 4A and 5). On the other hand, the percentage of cells positive for the studied proteins was still higher in the damaged white matter of CHIA than in CLIA MS lesions: BMP-2 $(23.77 \% \pm 8.10 \%$ versus $9.26 \% \pm 7.33 \%$; $P<0.05), \quad$ BMP-7 $(46.44 \% \pm 12.65 \%$ versus $29.57 \% \pm 5.66 \% ; P<0.05)$, BMPRII $(24.92 \% \pm 9.26 \%$ versus $14.78 \% \pm 9.21 \% ; P<0.05)$, and $\mathrm{pSMAD} 1 / 5 / 8$ $(12.84 \% \pm 4.93 \%$ versus $5.98 \% \pm 4.47 \% ; P<0.05)$. The differences were lost for BMP-4 when inflammatory activity was present in MS lesions; consistently, no differences were found in the percentage of cells stained with BMP-5 (Figures 4A and 5). Thus, the expression of BMPs and BMPRII is more important when the inflammatory activity of the chronic MS lesion is higher, whereas differences in the expression of the cytoplasmic mediator pSMAD $1 / 5 / 8$ were less pronounced.

In the NAGM proximal to the lesion, no differences were seen in the percentage of total cells expressing BMPs, BMPRII, or pSMAD1/5/8, except for BMP-7 in CIA compared with CHIA MS lesions $(21.85 \% \pm 3.64 \%$ versus $34.69 \% \pm 7.05 \%$, respectively; $P<0.05$ ) (Figure 4B), indicating that major changes occur in damaged white matter.

Expression of BMPs, BMPRII, and pSMAD1/5/8 in Lesions of Other White Matter-Damaging Diseases

Expression Pattern of BMP-2, BMP-4, BMP-5, BMP-7, BMPRII, and pSMAD1/5/8 in NNC, AI, and PML Lesions

The expression of the studied proteins was first characterized in NNC, PML, and AI in morphologically identified CNS populations. In the normal-appearing white matter of NNC, the expression of BMP-2, BMP-4, BMP-5, and BMP7, BMPRII, and pSMAD1/5/8 was detected in the oligodendrocytes, but none of them were detected in astrocytes or microglia/macrophages, probably because of the lack of damage in the tissue. In the damaged white matter of PML lesions, BMP-2 and BMP-5 were observed only in

Figure 3 Characterization of bone morphogenetic protein (BMP)-2, BMP-4, BMP-5, BMP-7, BMP type II receptor (BMPRII), and phosphorylated SMAD (pSMAD) $1 / 5 / 8$ in the gray matter of the different disease conditions studied. The expression of BMP-2, BMP-4, BMP-5, and BMP-7; BMPRII; and pSMAD1/5/8 was assessed in the gray matter adjacent to lesion in chronic multiple sclerosis (MS) lesion with high inflammatory activity (CHIA), chronic MS lesion with low inflammatory activity (CLIA), and chronic inactive (CIA) MS lesions, in acute infarct (AI) lesion and progressive multifocal leukoencephalopathy (PML) lesions and in the normal-appearing gray matter adjacent to the lesion in nonneurologic control (NNC). Different cell types were morphologically identified. Arrows indicate positive immunostaining, arrows with thick heads, neurons; arrows with thin heads, oligodendrocytes. Scale bar $=100 \mu \mathrm{m}$ (all images). Original magnification, $\times 40$ (all images). 
microglia/macrophages. BMP-4 and BMP-7 were detected not only in microglia/macrophages but also in astrocytes and oligodendrocytes, BMPRII was expressed in microglia/ macrophages and astrocytes, and pSMAD1/5/8 was observed mainly in microglia/macrophages and
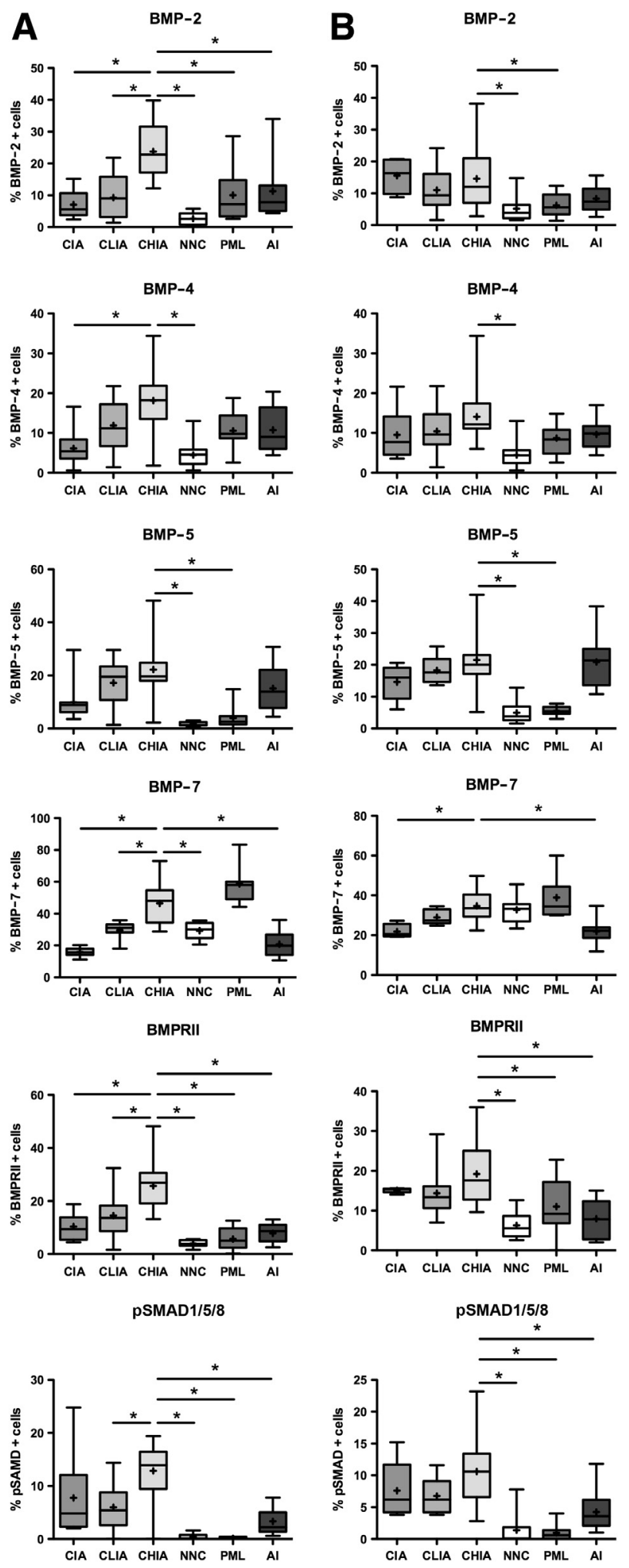

oligodendrocytes. In AI lesions, BMP-2, BMP-4, BMPRII, and pSMAD1/5/8 were observed in microglia/macrophages, BMP-5 was expressed in astrocytes, and BMP-7 was observed in the cytoplasm of oligodendrocytes (Figure 2).

Regarding the gray matter, neurons of the NAGM proximal to the lesion in PML and AI and NAGM in NNC expressed all of the examined proteins, similar to the pattern observed in MS lesions (Figure 3).

BMPs, BMPRII, and pSMAD1/5/8 Are Highly Expressed in Chronic Inflammatory Lesions of MS Compared with Lesions of Other White Matter-Damaging Diseases

It was determined whether the percentage of expression of the studied proteins was more relevant in MS lesions than in lesions of other diseases that cause damage in the white matter. CNS areas of NNC, AI, and PML lesions were immunostained, and the percentage of cells that expressed BMPs, BMPRII, and pSMAD1/5/8 were compared with that in CHIA MS lesions in both the normal-appearing white matter and the NAGM (in NNC) or the damaged white matter and NAGM proximal to the lesion (in AI, PML, CHIA MS lesions).

In the white matter, a higher percentage of cells that expressed BMP-2, BMP-4, BMP-5, and BMP-7; BMPRII; and pSMAD1/5/8 was observed in CHIA lesions than in the normal-appearing white matter of NNC (Figures 4A and 5), suggesting that the BMP signaling pathway is activated when the white matter is damaged. Moreover, BMP-2, BMPRII, and pSMAD1/5/8 were highly represented in CHIA lesions $(23.77 \% \pm 8.10 \%, 24.92 \% \pm 9.26 \%$, and $12.84 \% \pm 4.93 \%$ of cells stained for BMP2, BMPRII, and pSMAD1/5/8, respectively) compared with AI $(11.33 \% \pm 9.33 \%, 7.84 \% \pm 3.48 \%$, and $3.33 \% \pm 2.57 \%$, respectively; $P<0.05)$ and PML $(10.06 \% \pm 9.12 \%$, $5.67 \% \pm 4.18 \%$, and $0.14 \% \pm 0.19 \%$, respectively; $P<0.05$ ) (Figure 4A). The percentage of cells that

Figure 4 Differential expression of bone morphogenetic protein (BMP)2, BMP-4, BMP-5, BMP-7, BMP type II receptor (BMPRII), and phosphorylated SMAD (PSMAD) $1 / 5 / 8$ in multiple sclerosis (MS) lesions with different degree of inflammatory activity and in acute infarct (AI) lesion, progressive multifocal leukoencephalopathy (PML) lesion, and nonneurologic control (NNC). The expression of BMP-2, BMP-4, BMP-5, BMP-7, BMPRII, and PSMAD1/5/8 was quantified in the damaged white matter and normal-appearing gray matter of MS lesions, lesions from other demyelinating diseases, and the normal-appearing white and gray matter of nonneurologic patients. All conditions were compared with chronic MS lesion with high inflammatory activity (CHIA), applying Kruskal-Wallis test and, subsequently, Dunn test to evaluate the pairwise comparisons of CHIA MS lesions to all of the other conditions. Quantification in the white $(\mathbf{A})$ and gray (B) matter. The data are expressed as the percentage of positive cells over total nuclei. Box plots represent data from the first to the third quartile, bars represent the minimum and the maximum values, lines represent the median, and plus symbols represent the mean value. $n=21$ to 25 (A and $\mathbf{B}$, CHIA lesions); $n=6$ to 9 [A and $\mathbf{B}$, chronic MS lesion with low inflammatory activity (CLIA)]; $n=4$ to 8 [A and $\mathbf{B}$, chronic inactive (CIA) MS lesions]; $n=6$ to 9 (A and $\mathbf{B}, \mathrm{PML}$ lesions); $n=10$ to 12 (A and B, AI lesions); $n=9$ or 10 (A and $\mathbf{B}$, normal-appearing white and gray matter of nonneurologic patients). ${ }^{*} P<0.05$. 


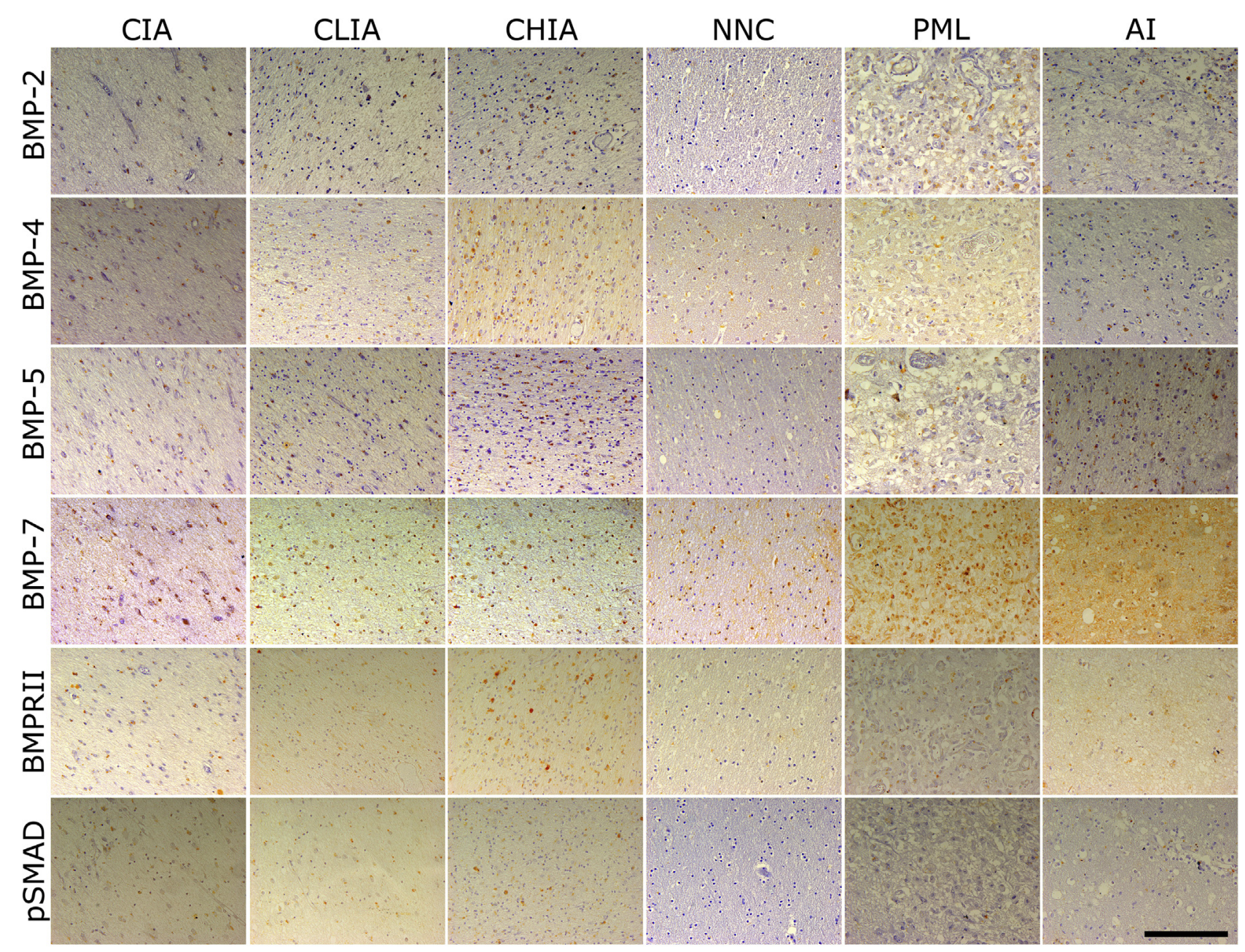

Figure 5 Quantification of bone morphogenetic protein (BMP)-2, BMP-4, BMP-5, BMP-7, BMP type II receptor (BMPRII), and phosphorylated SMAD (pSMAD) 1/5/8 expression in multiple sclerosis (MS) lesions and other demyelinating lesions. Micrographs show a general view of the expression of BMP-2, BMP-4, BMP-5, BMP-7, BMPRII, and pSMAD1/5/8 in the white matter of MS lesions [chronic with high inflammatory activity (CHIA) lesions, chronic with low inflammatory activity (CLIA) lesions, and chronic inactive (CIA) lesions], lesions from other demyelinating diseases [progressive multifocal leukoencephalopathy (PML) lesions and acute infarct (AI) lesions], and normal-appearing white matter [nonneurologic control (NNC)]. Scale bar $=200 \mu \mathrm{m}($ all images). Original magnification, $\times 20$ (all images).

expressed BMP-5 was significantly decreased in PML compared with CHIA MS lesions $(1.70 \% \pm 1.17 \%$ versus $21.83 \% \pm 9.81 \%$, respectively; $P<0.05$ ), and the percentage of BMP-7-positive cells was lower in AI damaged white matter than in CHIA MS lesions $(20.77 \% \pm 7.53 \%$ versus $46.44 \% \pm 12.65 \%$, respectively; $P<0.05)$ (Figures 4A and 5).

In the NAGM proximal to the lesion, a prominent expression of all of the studied proteins, except for BMP-7, was observed in CHIA lesions compared with NNC NAGM (Figure 4B). Similar to the results in the damaged white matter, the percentage of BMPRII- and pSMAD1/5/8positive cells was increased in CHIA lesions $(19.23 \% \pm 7.50 \%$ and $10.62 \% \pm 4.90 \%$, respectively) with respect to AI $(7.95 \% \pm 4.33 \%$ and $4.23 \% \pm 3.05 \%$, respectively; $P<0.05)$ or PML lesions $(11.00 \% \pm 7.04 \%$ and $0.97 \% \pm 1.42 \%$, respectively; $P<0.05$ ) (Figure $4 \mathrm{~B}$ ).
The percentage of BMP-2-positive cells was higher in CHIA MS lesions than in PML $(14.62 \% \pm 9.17 \%$ versus $6.23 \% \pm 3.76 \%$, respectively; $P<0.05$ ). In contrast, the percentage of BMP-4-stained cells was constant in all of the pathologic conditions (Figure 4B). Similar to the results obtained in the damaged white matter, the percentage of cells that expressed BMP-5 and BMP-7 in the NAGM proximal to the lesion differed between AI and PML lesions: BMP-5 was expressed in a lower percentage of cells in PML than in CHIA $(5.53 \% \pm 1.58 \%$ versus $20.90 \% \pm 8.83 \%$, respectively; $P<0.05$ ), and a lower percentage of BMP-7-positive cells was found in AI lesions compared with CHIA $(21.90 \% \pm 5.49 \%$ versus $34.69 \% \pm 7.50 \%$, respectively; $P<0.05$ ) (Figure 4B). The data indicate that although BMPs are differentially expressed in the gray matter when the white matter is damaged, changes in their expression are more relevant 
within the lesion than in the gray matter. Overall, the expression of BMPs, BMPRII, and pSMAD1/5/8 also increased in other demyelinating conditions. In addition, the increment of BMP expression was primarily observed in the damaged white matter and it was more pronounced in active MS lesions with high inflammatory activity.

\section{Discussion}

BMPs are serious candidates to have a role in MS pathogenesis because they are up-regulated in demyelinating conditions, ${ }^{20,21}$ they tightly control neural stem cell homeostasis,,${ }^{922,23}$ and they hinder remyelination and repair of the damaged CNS. ${ }^{8,24,25}$ In addition, BMPs also have a role in the regulation of the immune system; they regulate the activation and differentiation of certain immune cell populations (eg, regulatory $\mathrm{T}$ cells, $\mathrm{CD} 4^{+}$type 17 helper $\mathrm{T}$ cells, natural killer cells, and dendritic cells), they control the proliferation of $\mathrm{T}$ cells, and they have an impact in the immune function of a variety of cell types. ${ }^{26,27}$ For instance, whether they may be detrimental or beneficial in the context of MS is still unveiled. It probably depends on the target cell (eg, which BMPRs are expressed) and on the environment in the damaged tissue (eg, the presence of inhibitory signals and the composition of the extracellular matrix, among others).

Herein, we studied the expression of BMPs (BMP-2, BMP-4, BMP-5, and BMP-7) and the activation of the BMP canonical pathway by detecting BMPRII and the phosphorylated form of the cytoplasmic mediator SMAD1/5/8 in MS lesions. Oligodendrocytes expressed BMP-2 and BMP7, BMPRII, and pSMAD1/5/8, indicating that BMP signaling is active in these cell types. Astrocytes and microglia/macrophages within the lesion as well as neurons of the NAGM proximal to the lesion expressed all of the BMPs, BMPRII, and pSMAD1/5/8. These results expand our previous knowledge of the expression of BMPs in the CNS under pathologic conditions in both disease lesions and in animal models of MS..$^{20,21}$

It was confirmed that, when comparing MS lesions with different degrees of inflammatory activity, the major differences in the expression of BMPs resided in the damaged white matter and not in the NAGM proximal to the lesion. Thus, when the inflammatory activity in the lesion was higher, the expression of BMP-2, BMP-7, BMPRII, and pSMAD1/5/8 was higher. By contrast, BMP-4 and BMP-5 remained elevated in all MS lesions (CHIA, CLIA, and CIA). It is reported that immune cells express BMPs ${ }^{28}$ and that the expression of BMPs in the peripheral blood mononuclear cells of relapsing-remitting MS patients is deregulated and increased, especially in monocytes and $\mathrm{T}$ cells, with respect to healthy controls. ${ }^{29}$ The correlation of the increased BMP signaling pathway (pSMAD1/5/8 staining) with a higher inflammatory activity in MS lesions may be attributed in part to the contribution of inflammatory infiltrates to the expression of the proteins. Thus, we hypothesize that the immune cells that are infiltrating the CNS in MS lesions would be promoting a negative environment for tissue repair, although this issue has not been addressed in the present work. So, further studies should be conducted to unveil the expression of BMPs in the inflammatory infiltrate and to figure out the biological relevance of immune cell-derived BMPs in the context of MS lesions.

The expression of the aforementioned proteins was compared in the damaged white matter and NAGM proximal to the lesion of CHIA MS lesions with that in normalappearing white and gray matter of NNC. All BMPs and the signaling pathway were found to be elevated in the diseased condition, consistent with the findings of Deininger and collaborators. ${ }^{20}$ It was next determined if the pattern of expression was exclusive of CHIA MS lesions by comparing the expression of BMPs, BMPRII, and pSMAD1/5/8 with that in lesions of other white matter-damaging diseases, such as AI and PML. It could not be concluded that the BMP increase was solely found in MS lesions; in fact, BMP expression has been previously reported to be up-regulated in different demyelinating conditions and models. ${ }^{8,20,30}$ However, there was expression of pSMAD1/5/8 in MS lesions, whereas it was rarely detected in the damaged white matter and NAGM proximal to the lesion in AI and PML, indicating that BMP signaling is probably more active in MS lesions. Similarly, in myelin oligodendrocyte glycoprotein-induced experimental autoimmune encephalomyelitis, an animal model for MS, BMP4, BMP-6, and BMP-7 were up-regulated on induction of the disease. ${ }^{21}$ Remyelination failure is one of the keystones of MS research. Although approximately $40 \%$ of active MS lesions remyelinate, translating into functional axon recovery, this process is incomplete and heterogeneous between MS patients. ${ }^{31}$ Remyelination directly correlates with the recruitment of OPCs into demyelinated areas; nevertheless, in approximately $70 \%$ of chronic lesions of MS, premyelinating oligodendrocytes are detected but are unable to remyelinate axons. ${ }^{15}$ Why remyelination fails is still unknown, although different molecules may be involved in the impairment of myelin repair by inhibiting the recruitment of OPCs into the lesion (eg, semaphorin $3 \mathrm{~A}^{14}$ ), inhibiting the differentiation and maturation of OPCs into myelinating oligodendrocytes (eg, $\operatorname{NogoA}^{32}$ ), or hindering the predisposition of chronically demyelinated axons to be remyelinated. ${ }^{15}$ Moreover, BMPs have been largely described as promoters of astrogliogenesis ${ }^{6}$ and are also important in the regulation of the differentiation of oligodendrocytes, inhibiting their maturation into myelinating oligodendrocytes. Taking into account that OPCs in the edge of chronic lesions are often unable to migrate and/or maturate into myelinating oligodendrocytes, ${ }^{14,33}$ and that demyelinated axons have probably changed their molecular composition in such a way that they are not prone to remyelinate, ${ }^{15}$ we hypothesize that the increased expression of BMPs, BMPRII, and pSMAD1/5/8 in MS lesions probably 
contributes to a poor remyelination of the damaged white matter. Nonetheless, to further corroborate this last statement, more detailed studies would be desirable to determine the oligodendroglial lineage stage of the Olig2-positive cells within the lesion. In addition, an increase in the expression of BMP-4 and BMP-7 in a context of demyelination favored gliosis. It has also been associated with an up-regulation in chondroitin sulfate proteoglycans, which are important components of the glial scar and further impede the regeneration of axons into the lesion. ${ }^{34}$ Altogether, these actions would generate a negative environment within the lesion that would ultimately impair repair of the damaged area. Of note is the increased expression of BMPs, BMPRII, and pSMAD1/5/8 in the NAGM proximal to the lesion, indicating that demyelination also affects the pattern of expression in the tissue distal to the lesion, probably affecting other processes, such as neurogenesis, ${ }^{9}$ or making axons less prone to be remyelinated. ${ }^{15}$

In this study, we describe the expression of BMPs, BMPRII, and pSMAD1/5/8 in chronic inflammatory MS lesions and their positive correlation with the inflammatory activity in the lesion. The results suggest that BMPs may have a role in demyelinating diseases; nevertheless, it has to be taken into account that these studies were performed in human post-mortem tissue. So, it is not possible to find out the dynamics of BMP expression in MS, which clearly would give us more relevant information of the involvement of BMPs in the disease. Nonetheless, this study opens the field to functional and preclinical studies to further elucidate whether BMPs play a role in MS pathogenesis, to determine whether they are beneficial or detrimental for the disease, and to explore the possibility of using BMPs as possible diagnostic and/or prognostic biomarkers for MS.

\section{Supplemental Data}

Supplemental material for this article can be found at https://doi.org/10.1016/j.ajpath.2018.11.007.

\section{References}

1. Mueller TD: Mechanisms of BMP-receptor interaction and activation. Vitam Horm 2015, 99:1-61

2. Wieser R, Wrana JL, Massague J: GS domain mutations that constitutively activate $\mathrm{T}$ beta R-I, the downstream signaling component in the TGF-beta receptor complex. EMBO J 1995, 14:2199-2208

3. Nohe A, Hassel S, Ehrlich M, Neubauer F, Sebald W, Henis YI, Knaus P: The mode of bone morphogenetic protein (BMP) receptor oligomerization determines different BMP-2 signaling pathways. J Biol Chem 2002, 277:5330-5338

4. Massague J, Seoane J, Wotton D: Smad transcription factors. Genes Dev 2005, 19:2783-2810

5. Liu A, Niswander LA: Bone morphogenetic protein signalling and vertebrate nervous system development. Nat Rev Neurosci 2005, 6: 945-954

6. Mabie PC, Mehler MF, Marmur R, Papavasiliou A, Song Q, Kessler JA: Bone morphogenetic proteins induce astroglial differentiation of oligodendroglial-astroglial progenitor cells. J Neurosci 1997, 17:4112-4120

7. Grinspan JB, Edell E, Carpio DF, Beesley JS, Lavy L, Pleasure D, Golden JA: Stage-specific effects of bone morphogenetic proteins on the oligodendrocyte lineage. J Neurobiol 2000, 43:1-17

8. Sabo JK, Aumann TD, Merlo D, Kilpatrick TJ, Cate HS: Remyelination is altered by bone morphogenic protein signaling in demyelinated lesions. J Neurosci 2011, 31:4504-4510

9. Mira H, Andreu Z, Suh H, Lie DC, Jessberger S, Consiglio A, San Emeterio J, Hortiguela R, Marques-Torrejon MA, Nakashima K, Colak D, Gotz M, Farinas I, Gage FH: Signaling through BMPR-IA regulates quiescence and long-term activity of neural stem cells in the adult hippocampus. Cell Stem Cell 2010, 7:78-89

10. Sospedra M, Martin R: Immunology of multiple sclerosis. Annu Rev Immunol 2005, 23:683-747

11. Tallantyre EC, Bo L, Al-Rawashdeh O, Owens T, Polman $\mathrm{CH}$, Lowe JS, Evangelou N: Clinico-pathological evidence that axonal loss underlies disability in progressive multiple sclerosis. Mult Scler 2010, 16:406-411

12. Patani R, Balaratnam M, Vora A, Reynolds R: Remyelination can be extensive in multiple sclerosis despite a long disease course. Neuropathol Appl Neurobiol 2007, 33:277-287

13. Patrikios P, Stadelmann C, Kutzelnigg A, Rauschka H, Schmidbauer M, Laursen H, Sorensen PS, Bruck W, Lucchinetti C, Lassmann H: Remyelination is extensive in a subset of multiple sclerosis patients. Brain 2006, 129:3165-3172

14. Boyd A, Zhang H, Williams A: Insufficient OPC migration into demyelinated lesions is a cause of poor remyelination in MS and mouse models. Acta Neuropathol 2013, 125:841-859

15. Chang A, Tourtellotte WW, Rudick R, Trapp BD: Premyelinating oligodendrocytes in chronic lesions of multiple sclerosis. N Engl J Med 2002, 346:165-173

16. Franklin RJ: Why does remyelination fail in multiple sclerosis? Nat Rev Neurosci 2002, 3:705-714

17. Costa C, Martinez-Saez E, Gutierrez-Franco A, Eixarch H, Castro Z, Ortega-Aznar A, Ramon YCS, Montalban X, Espejo C: Expression of semaphorin $3 \mathrm{~A}$, semaphorin 7A and their receptors in multiple sclerosis lesions. Mult Scler 2015, 21:1632-1643

18. Siegel S, Castellan NJ: Nonparametric Statistics for the Behavioral Sciences. New York, NY, MacGraw Hill Int, 1988

19. R Core Team RCT: R: A Language and Environment for Statistical Computing. R 3.2.3 software ed.: Vienna, Austria, R Foundation for Statistical Computing, 2015

20. Deininger M, Meyermann R, Schluesener H: Detection of two transforming growth factor-beta-related morphogens, bone morphogenetic proteins-4 and -5 , in RNA of multiple sclerosis and Creutzfeldt-Jakob disease lesions. Acta Neuropathol 1995, 90:76-79

21. Ara J, See J, Mamontov P, Hahn A, Bannerman P, Pleasure D, Grinspan JB: Bone morphogenetic proteins 4, 6, and 7 are up-regulated in mouse spinal cord during experimental autoimmune encephalomyelitis. J Neurosci Res 2008, 86:125-135

22. Coskun V, Venkatraman G, Yang H, Rao MS, Luskin MB: Retroviral manipulation of the expression of bone morphogenetic protein receptor Ia by SVZa progenitor cells leads to changes in their p19(INK4d) expression but not in their neuronal commitment. Int J Dev Neurosci 2001, 19:219-227

23. Colak D, Mori T, Brill MS, Pfeifer A, Falk S, Deng C, Monteiro R, Mummery C, Sommer L, Gotz M: Adult neurogenesis requires Smad4mediated bone morphogenic protein signaling in stem cells. J Neurosci 2008, 28:434-446

24. See JM, Grinspan JB: Sending mixed signals: bone morphogenetic protein in myelination and demyelination. J Neuropathol Exp Neurol 2009, 68:595-604

25. Chen LP, Li ZF, Ping M, Li R, Liu J, Xie XH, Song XJ, Guo L: Regulation of Olig2 during astroglial differentiation in the subventricular zone of a cuprizone-induced demyelination mouse model. Neuroscience 2012, 221:96-107 
26. Eixarch H, Calvo-Barreiro L, Montalban X, Espejo C: Bone morphogenetic proteins in multiple sclerosis: role in neuroinflammation. Brain Behav Immun 2018, 68:1-10

27. Chen W, Ten Dijke P: Immunoregulation by members of the TGFbeta superfamily. Nat Rev Immunol 2016, 16:723-740

28. Martinez VG, Sacedon R, Hidalgo L, Valencia J, FernandezSevilla LM, Hernandez-Lopez C, Vicente A, Varas A: The BMP pathway participates in human naive CD4+ T cell activation and homeostasis. PLoS One 2015, 10:e0131453

29. Mausner-Fainberg K, Urshansky N, Regev K, Auriel E, Karni A: Elevated and dysregulated bone morphogenic proteins in immune cells of patients with relapsing-remitting multiple sclerosis. J Neuroimmunol 2013, 264:91-99

30. Cate HS, Sabo JK, Merlo D, Kemper D, Aumann TD, Robinson J, Merson TD, Emery B, Perreau VM, Kilpatrick TJ: Modulation of bone morphogenic protein signalling alters numbers of astrocytes and oligodendroglia in the subventricular zone during cuprizone-induced demyelination. J Neurochem 2010, 115:11-22

31. Franklin RJ, Ffrench-Constant C: Remyelination in the CNS: from biology to therapy. Nat Rev Neurosci 2008, 9:839-855

32. Mi S, Miller RH, Tang W, Lee X, Hu B, Wu W, Zhang Y, Shields CB, Zhang Y, Miklasz S, Shea D, Mason J, Franklin RJ, Ji B, Shao Z, Chedotal A, Bernard F, Roulois A, Xu J, Jung V, Pepinsky B: Promotion of central nervous system remyelination by induced differentiation of oligodendrocyte precursor cells. Ann Neurol 2009, 65:304-315

33. Kuhlmann T, Miron V, Cui Q, Wegner C, Antel J, Bruck W: Differentiation block of oligodendroglial progenitor cells as a cause for remyelination failure in chronic multiple sclerosis. Brain 2008, 131: $1749-1758$

34. Fuller ML, DeChant AK, Rothstein B, Caprariello A, Wang R, Hall AK, Miller RH: Bone morphogenetic proteins promote gliosis in demyelinating spinal cord lesions. Ann Neurol 2007, 62:288-300 